\title{
The New Operators in Topological Space
}

\author{
NebojŠA Elez ANd OGnjen PAPAZ
}

\begin{abstract}
In this paper some of the properties of the boundary operator were proved and the way we can define topology on some set $X$ using the boundary operator was shown. Then, we examined the properties of the two new operators which we defined here and also we showed how we can define topology on some set $X$ using any of these new operators.
\end{abstract}

\section{INTRODUCTION}

For a subset $A$ of a topological space $X$ we write $\bar{A}, A^{\circ}, \operatorname{Fr} A$ for the closure, interior and boundary of a set $A$ respectively.

We'll use the following assertion, which is proved in [1],

$$
\operatorname{Fr}(A \cup B) \subseteq \operatorname{Fr} A \cup \operatorname{Fr} B .
$$

We define the operator ${ }^{+}$on a topological space $X$ in the following way: for a subset $A$ of $X, A^{+}=\bar{A} \backslash A$.

We define the operator ${ }^{*}$ on a topological space $X$ in the following way: for a subset $A$ of $X, A^{*}=A \backslash A^{\circ}$.

\section{MAIN RESUlts}

Theorem 1. In every topological space $X$, the following conditions hold:

1) $\operatorname{Fr} \emptyset=\emptyset$;

2) $\operatorname{Fr} A \cup \operatorname{Fr}(B)=(A \cap \operatorname{Fr} B) \cup \operatorname{Fr}(A \cup B) \cup(\operatorname{Fr} A \cap B)$;

3) $\operatorname{Fr}(\operatorname{Fr} A) \subseteq \operatorname{Fr} A$;

4) $\operatorname{Fr}(X \backslash A)=\operatorname{Fr} A$.

If an operator $\varphi$ on some set $X$ satisfies the conditions 1)-4), then the operator $\mathrm{k}$ defined in the following way: $\mathrm{k}(A)=A \cup \varphi(A)$, is a closure operator on $X$, and $\varphi(A)=\operatorname{Fr} A$ for every subset $A$ of a space $X$, in which the topology is induced by $\mathrm{k}$.

2000 Mathematics Subject Classification. Primary: 54A10.

Key words and phrases. Topological operators. 
Remark 1. Similar but different conditions were given in [2], namely there were five conditions and it was shown that topology can be defined using them.

Proof. Conditions 1) and 4) holds trivially.

Lets prove 2). From (1) it easily follows that

$$
(A \cap \operatorname{Fr} B) \cup \operatorname{Fr}(A \cup B) \cup(\operatorname{Fr} A \cap B) \subseteq \operatorname{Fr} A \cup \operatorname{Fr} B .
$$

Because of the symmetry it remains to be proven that

$\operatorname{Fr} A \subseteq(A \cap \operatorname{Fr} B) \cup \operatorname{Fr}(A \cup B) \cup(\operatorname{Fr} A \cap B)$.

$1^{\circ}$ Let $x \in \operatorname{Fr} A \cap \operatorname{Fr} B, x \in X \backslash A$, and $x \in X \backslash B$.

It's easy to conclude that $x \in \overline{A \cup B}$ and $x \in X \backslash(A \cup B)^{\circ}$ i.e. $x \in \operatorname{Fr}(A \cup B)$.

$2^{\circ}$ Let $x \in \operatorname{Fr} A \backslash \operatorname{Fr} B, x \in X \backslash B$.

Then, $x \in X \backslash(A \cup B)^{\circ}$ because otherwise we would have that $x \in A^{\circ}$, which is impossible.

Hence, $x \in \operatorname{Fr}(A \cup B)$.

3) $\operatorname{Fr}(\operatorname{Fr} A) \subseteq \overline{\operatorname{Fr} A}=\operatorname{Fr} A$.

Now, let the operator $\varphi$ on some set $X$ satisfies conditions 1)-4). For operator k, defined with $\mathrm{k}(A)=A \cup \varphi(A)$, we have:

1) $\mathrm{k}(\emptyset)=\emptyset \cup \varphi(\emptyset)=\emptyset$;

2) $A \subseteq A \cup \varphi(A)=\mathrm{k}(A)$

3) $\mathrm{k}(\mathrm{k}(A))=\mathrm{k}(A \cup \varphi(A))=A \cup \varphi(A) \cup \varphi(A \cup \varphi(A)) \subseteq$

$A \cup \varphi(A) \cup \varphi(A) \cup \varphi(\varphi(A)) \subseteq A \cup \varphi(A) \cup \varphi(A)=\mathrm{k}(A) ;$

4) $\mathrm{k}(A \cup B)=A \cup B \cup \varphi(A \cup B) \subseteq$

$A \cup B \cup \varphi(A) \cup \varphi(B)=\mathrm{k}(A) \cup \mathrm{k}(B)$ and

$\mathrm{k}(A) \cup \mathrm{k}(B)=A \cup \varphi(A) \cup B \cup \varphi(B)=$

$A \cup B \cup(A \cap \varphi(B)) \cup \varphi(A \cup B) \cup(B \cap \varphi(A)) \subseteq$

$A \cup B \cup \varphi(A \cup B)=\mathrm{k}(A \cup B)$.

Thus, we have proven that $\mathrm{k}$ is indeed the closure operator on $X$.

As for the last part, for every subset $A$ of $X$ we have

$$
\text { Fr } \begin{aligned}
A & =\mathrm{k}(A) \cap \mathrm{k}(X \backslash A) \\
& =(A \cup \varphi(A)) \cap((X \backslash A) \cup \varphi(X \backslash A)) \\
& =(\varphi(A) \cap(X \backslash A)) \cup(A \cap \varphi(A)) \cup \varphi(A) \\
& =\varphi(A) .
\end{aligned}
$$

Theorem 2. Let $A$ and $B$ be the subsets of a topological space $X$, and let

$$
\begin{aligned}
& \tau=\{\operatorname{Fr} A, \operatorname{Fr} B, \operatorname{Fr}(A \Delta B)\} \text { and } \\
& \xi=\{\operatorname{Fr}(A \cup B), \operatorname{Fr}(A \cap B), \operatorname{Fr}(A \backslash B), \operatorname{Fr}(B \backslash A)\} .
\end{aligned}
$$


The union of any two distinct elements of $\tau$ is equal to the union of any three distinct elements of $\xi$. (Here $\Delta$ stands for the symmetric difference.)

Proof. Firstly, we will show that $\operatorname{Fr} A \cup \operatorname{Fr} B=\operatorname{Fr}(A \backslash B) \cup \operatorname{Fr}(A \cap B) \cup \operatorname{Fr}(B \backslash A)$.

$$
\begin{aligned}
\operatorname{Fr}(A \cap B) & =\operatorname{Fr}(X \backslash(A \cap B)) \\
& =\operatorname{Fr}((X \backslash A) \cup(X \backslash B)) \subseteq \operatorname{Fr}(X \backslash A) \cup \operatorname{Fr}(X \backslash B) \\
& =\operatorname{Fr} A \cup \operatorname{Fr} B . \\
\operatorname{Fr}(A \backslash B) & =\operatorname{Fr}(A \cap(X \backslash B)) \subseteq \operatorname{Fr} A \cup \operatorname{Fr} B .
\end{aligned}
$$

Analogously,

$$
\operatorname{Fr}(B \backslash A) \subseteq \operatorname{Fr} A \cup \operatorname{Fr} B
$$

So,

$$
\operatorname{Fr}(A \backslash B) \cup \operatorname{Fr}(A \cap B) \cup \operatorname{Fr}(B \backslash A) \subseteq \operatorname{Fr} A \cup \operatorname{Fr} B .
$$

On the other hand, we have

$$
\begin{aligned}
\operatorname{Fr} A \cup \operatorname{Fr} B & =\operatorname{Fr}((A \backslash B) \cup(A \cap B)) \cup \operatorname{Fr}((B \backslash A) \cup(A \cap B)) \\
& \subseteq \operatorname{Fr}(A \backslash B) \cup \operatorname{Fr}(A \cap B) \cup \operatorname{Fr}(B \backslash A) .
\end{aligned}
$$

So we have proved (1).

If we put $X \backslash B$ in (1) instead of $B$, we get

Fr $A \cup \operatorname{Fr} B=\operatorname{Fr}(A \backslash B) \cup \operatorname{Fr}(A \cap B) \cup \operatorname{Fr}(A \cup B)$.

If we put $X \backslash A$ in (1) instead of $A$ we get

Fr $A \cup \operatorname{Fr} B=\operatorname{Fr}(A \backslash B) \cup \operatorname{Fr}(A \cap B) \cup \operatorname{Fr}(B \backslash A)$.

If we put $X \backslash A$ instead of $A$ and $X \backslash B$ instead of $B$ in (1), we get

$$
\text { Fr } A \cup \operatorname{Fr} B=\operatorname{Fr}(A \cup B) \cup \operatorname{Fr}(A \cap B) \cup \operatorname{Fr}(B \backslash A) .
$$

According to what we have proven so far it suffices to prove that

$$
\begin{aligned}
\operatorname{Fr} A \cup \operatorname{Fr}(A \Delta B) & =\operatorname{Fr}(A \backslash B) \cup \operatorname{Fr}(A \cap B) \cup \operatorname{Fr}(B \backslash A) \\
& =\operatorname{Fr} B \cup \operatorname{Fr}(A \Delta B) .
\end{aligned}
$$

From (2) we have that

$$
\begin{aligned}
\operatorname{Fr} A \cup \operatorname{Fr}(A \Delta B) & =\operatorname{Fr}(A \backslash(A \Delta B)) \cup \operatorname{Fr}(A \cap(A \Delta B)) \cup \operatorname{Fr}((A \Delta B) \backslash A) \\
& =\operatorname{Fr}(A \cap B) \cup \operatorname{Fr}(A \backslash B) \cup \operatorname{Fr}(B \backslash A) \\
& =\operatorname{Fr} B \cup \operatorname{Fr}(A \Delta B) .
\end{aligned}
$$

Theorem 3. In every topological space $X$, the following conditions hold:

1) $\emptyset^{+}=\emptyset$;

2) $A \cap A^{+}=\emptyset$; 
3) $(A \cup B)^{+}=\left(A^{+} \backslash B\right) \cup\left(B^{+} \backslash A\right)$;

4) $\left(A^{+}\right)^{+} \subseteq A$.

If an operator $\varphi$ on some set $X$ satisfies the above conditions 1)-4), then the operator $\mathrm{k}$, defined in the following way: $\mathrm{k}(A)=A \cup \varphi(A)$, is a closure operator on $X$, and $\varphi(A)=A^{+}$for every subset $A$ of a space $X$, in which the topology is induced by $\mathrm{k}$.

Proof. Conditions 1) and 2) holds trivially.

As for the third and fourth we have:

$$
\begin{aligned}
(A \cup B)^{+} & =\overline{A \cup B} \backslash(A \cup B) \\
& =((\bar{A} \backslash A) \backslash B) \cup((\bar{B} \backslash B) \backslash A) \\
& =\left(A^{+} \backslash B\right) \cup\left(B^{+} \backslash A\right) . \\
\left(A^{+}\right)^{+} & =\overline{A^{+}} \backslash A^{+} \subseteq \bar{A} \backslash(\bar{A} \backslash A) \subseteq A .
\end{aligned}
$$

Now, let an operator $\varphi$ on some set $X$ satisfies the above conditions 1)-4). For the operator $\mathrm{k}$, defined with $\mathrm{k}(A)=A \cup \varphi(A)$, we have:

1) $\bar{\emptyset}=\emptyset \cup \varphi(\emptyset)=\emptyset$;

2) $A \subseteq A \cup \varphi(A)=\mathrm{k}(A)$;

3) $\mathrm{k}(A \cup B)=(A \cup B) \cup \varphi(A \cup B)=(A \cup B) \cup(\varphi(A) \backslash B) \cup(\varphi(B) \backslash A)$ $=A \cup \varphi(A) \cup B \cup \varphi(B)=\mathrm{k}(A) \cup \mathrm{k}(B)$;

4) $\mathrm{k}(\mathrm{k}(A))=\mathrm{k}(A) \cup \varphi(\mathrm{k}(A))=\mathrm{k}(A) \cup \varphi(A \cup \varphi(A))$ $=\mathrm{k}(A) \cup(\varphi(A) \backslash \varphi(A)) \cup(\varphi(\varphi(A)) \backslash A)=\mathrm{k}(A)$.

Thus, the operator $\mathrm{k}$ is a closure operator on $X$.

As for the last part, for every subset $A$ of $X$ we have

$$
A^{+}=\mathrm{k}(A) \backslash A=(A \cup \varphi(A)) \backslash A=\varphi(A) .
$$

Theorem 4. For every subset $A$ of a topological space $X$, the following conditions hold:

1) $\bar{A}=A \cup A^{+}$;

2) $A^{+}=\operatorname{Fr} A \backslash A$;

3) $\operatorname{Fr} A=A^{+} \cup(X \backslash A)^{+}$;

4) $A^{+} \cup B^{+}=\left(A \cap B^{+}\right) \cup(A \cup B)^{+} \cup\left(A^{+} \cap B\right)$;

5) $\bar{A}^{+}=\emptyset$;

6) $A$ is open if and only if $A^{+}=\operatorname{Fr} A$;

7) $A^{d}=\bigcup_{B \subseteq A} B^{+}$and $A^{+}=A^{d} \backslash A$, where $A^{d}$ is the set of density points of $\bar{A}$.

Proof. We will prove some of the assertions, others hold trivially.

4) Firstly, we'll show that the set on the LHS is subset of the set on the RHS. 
It suffices to prove that $A^{+} \backslash B \subseteq(A \cup B)^{+}$, which is equivalent with $(\bar{A} \backslash A) \backslash B \subseteq \overline{A \cup B} \backslash(A \cup B)$, and this is equivalent with $\bar{A} \subseteq \overline{A \cup B}$.

Now we'll show that the reversed inclusion holds. It suffices to prove that $(A \cup B)^{+} \subseteq A^{+} \cup B^{+}$. This is equivalent with $\overline{A \cup B} \backslash(A \cup B) \subseteq$ $(\bar{A} \backslash A) \cup(\bar{B} \backslash B)$ which is obviously true.

7) $B \subseteq A \Rightarrow B^{+}=\bar{B} \backslash B \subseteq B^{d} \subseteq A^{d} . x \in A^{d} \Rightarrow x \in \overline{A \backslash\{x\}} \Rightarrow x \in$ $\overline{A \backslash\{x\}} \backslash(A \backslash\{x\})=(A \backslash\{x\})^{+}=B^{+}$,

for $B \subseteq A$.

$A^{+}=\overline{\bar{A}} \backslash A=\left(A \cup A^{d}\right) \backslash A=A^{d} \backslash A$.

Let $\mathcal{C}_{1}$ be the family of all closed subsets of a topological space $X$. For $n \in \mathbb{N}$ let $\mathcal{C}_{n+1}=\left\{F \backslash B \mid F \in \mathcal{C}_{1}\right.$ and $\left.B \in \mathcal{C}_{n}\right\}$. Note that $A \in \mathcal{C}_{n}$ if and only if

$$
A=F_{1} \backslash\left(F_{2} \backslash \ldots\left(F_{n-1} \backslash F_{n}\right) \ldots\right),
$$

for some sequence $F_{1}, F_{2}, \ldots, F_{n}$ of the closed subsets of $X$.

Theorem 5. In every topological space $X$, the following holds: $A \in \mathcal{C}_{n}$ if and only if $A^{+^{n}}=\emptyset$. (We define $A^{+^{n}}$ inductively, $A^{+^{1}}=A^{+}$and $A^{+^{n+1}}=$ $\left(A^{+^{n}}\right)^{+}$.)

Proof. We'll prove that $A \in \mathcal{C}_{n} \Rightarrow A^{+^{n}}=\emptyset$ by induction.

For $n=1$ statement obviously holds. Let's assume that the statement holds for some natural number $n$. Let $A \in \mathcal{C}_{n+1}$. Then $A=F \backslash B$, where $F$ is closed and $B \in \mathcal{C}_{n}$. We have that

$$
A^{+}=\overline{F \backslash B} \backslash(F \backslash B)=(\overline{F \backslash B} \backslash F) \cup(\overline{F \backslash B} \cap B)=\overline{F \backslash B} \cap B \in \mathcal{C}_{n} .
$$

Hence, $A^{+^{n+1}}=\left(A^{+}\right)^{+^{n}}=\emptyset$.

Now let's prove the converse. If $A^{+^{n+1}}=\emptyset$ then $\left(A^{+}\right)^{+^{n}}=\emptyset$, and for $B=$ $A^{+}$we have that $A=\bar{A} \backslash B \in \mathcal{C}_{n+1}$ because $\bar{A}$ is closed and $B \in \mathcal{C}_{n+1}$.

Corollary 1. Following two statements are immediate consequences of the preceding theorem:

1) $A$ is closed if and only if $A^{+}=\emptyset$;

2) $A$ is locally closed i.e. difference of two closed sets if and only if $A^{+^{2}}=\emptyset$.

Remark 2. The authors are not certain whether or not $\bigcup_{n=1}^{+\infty} \mathcal{C}_{n}$ is equal to the algebra on a set $X$, generated by its topology.

Theorem 6. In every topological space $X$, the following conditions hold:

1) $X^{*}=\emptyset$;

2) $A^{*} \subseteq A$;

3) $(A \cap B)^{*}=\left(A^{*} \cap B\right) \cup\left(A \cap B^{*}\right)$;

4) $\left(A^{*}\right)^{*}=A^{*}$. 
If an operator $\varphi$ on some set $X$ satisfies the above conditions 1)-4), then the operator Int defined in the following way: $\operatorname{Int}(A)=A \backslash \varphi(A)$, is an interior operator on $X$, and $\varphi(A)=A^{*}$ for every subset $A$ of a space $X$, in which the topology is induced by Int.

Proof. Conditions 1) and 2) hold trivially.

3) $(A \cap B)^{*}=(A \cap B) \backslash(A \cap B)^{\circ}=(A \cap B) \cap\left(X \backslash\left(A^{\circ} \cap B^{\circ}\right)\right)=$ $\left(A \cap\left(X \backslash A^{\circ}\right) \cap B\right) \cup\left(A \cap B \cap\left(X \backslash B^{\circ}\right)\right)=\left(A^{*} \cap B\right) \cup\left(A \cap B^{*}\right)$.

4) $\left(A^{*}\right)^{*}=\left(A \backslash A^{\circ}\right) \backslash\left(A \backslash A^{\circ}\right)^{\circ}=A \backslash A^{\circ}=A^{*}$.

Now, let the operator $\varphi$ on some set $X$ satisfies the above conditions 1)-4). For operator Int, defined with $\operatorname{Int}(A)=A \backslash \varphi(A)$, we have:

1) $\operatorname{Int}(X)=X \backslash \varphi(X)=X$

2) $\operatorname{Int}(A)=A \backslash \varphi(A) \subseteq A$

3) $\operatorname{Int}(A \cap B)=(A \cap B) \backslash \varphi(A \cap B)=(A \cap B) \backslash((\varphi(A) \cap B) \cup(A \cap \varphi(B))=$ $(A \backslash \varphi(A)) \cap(B \backslash \varphi(B))=\operatorname{Int}(A) \cap \operatorname{Int}(B)$.

4) $\operatorname{Int}(\operatorname{Int}(A))=(A \backslash \varphi(A)) \backslash \varphi(A \backslash \varphi(A)) \supseteq$ $(A \backslash \varphi(A)) \backslash(\varphi(A) \cap \varphi(X \backslash \varphi(A))) \supseteq$ $(A \backslash \varphi(A)) \backslash(\varphi(A) \cap(X \backslash \varphi(A)))=\operatorname{Int}(A)$.

Thus, we have proven that Int is an interior operator on $X$.

As for the last part, for every subset $A$ of $X$ we have

$$
A^{*}=A \backslash \operatorname{Int}(A)=A \backslash(A \backslash \varphi(A))=\varphi(A) \cap A=\varphi(A) .
$$

\title{
REFERENCES
}

[1] Ryszard Engelking, General Topology, PWN Warszava, 1977.

[2] Nebojša Elez, Equations related to the boundary operator in topological space, Zb. Rad. (Kragujevac) No. 18(1996), 5-8.

\author{
Nebojs̃a Elez \\ Faculty of Philosophy \\ Alekse ŠAntića 1 \\ 71420 PALE \\ Bosnia ANd Herzegovina \\ E-mail address: nelez@ffuis.edu.ba \\ OgnJen Papaz \\ Faculty of Philosophy \\ Alekse ŠAntića 1 \\ 71420 PALE \\ Bosnia AND HERzEgovina \\ E-mail address: opapaz@ffuis.edu.ba
}

